\title{
CDISC SDTM AJCC Tumor Grade Response Terminology
}

National Cancer Institute

\section{Source}

National Cancer Institute. CDISC SDTM AJCC Tumor Grade Response Terminology. NCI Thesaurus. Code C135012.

Terminology associated with the AJCC tumor grade response codelist of the Clinical Data Interchange Standards Consortium (CDISC) Study Data T abulation Model (SDT M). 